\title{
Decision Support in Engineering Multivariable Control Systems for Unstable Objects
}

\author{
Bariy Ilyasov \\ Ufa State Aviation Technical \\ University \\ Ufa, Russia \\ ilyasov@tc.ugatu.ac.ru
}

\author{
Guzel Saitova \\ Ufa State Aviation Technical \\ University \\ Ufa, Russia \\ saitova@tc.ugatu.ac.ru
}

\author{
Elena Khalikova \\ Ufa State Aviation Technical \\ University \\ Ufa, Russia \\ khalikova_elena@mail.ru
}

\begin{abstract}
The problem of ensuring the stability of a multivariable automatic control system which consists of statically and dynamically unstable objects must be managed when solving applied problems. In theoretical terms, this problem is worked out poorly and there are no practical recommendations for its solution. Examples of unstable objects exist in different areas of knowledge. In mechanics, it is a double inverted pendulum, which itself is a biconnected system, or an interconnected system of several inverted pendulums. In the technique - gas turbine engine operation in some operating modes, control of a statically unstable aircraft. In social systems - the behavior of a group of people or groups in critical situations. In large-scale structures (socio-economic, ecological, etc.) there are always statically and dynamically unstable subsystems. For example, dynamic instability may occur when the operating conditions change, the connection is broken etc. Static instability manifests itself in some modes of operation of power plants. The paper analyzes the solution of this problem in the class of multivariable selforganizing systems, which form the structure of relations between unstable subsystems.
\end{abstract}

Keywords - unstable objects, multivariable automatic control system, complex technical object, frequency method, synthesis of communications, characteristic equation, statically and dynamically unstable objects

\section{INTRODUCTION}

The need to create multivariable systems arises when solving various problems of engineering control systems of complex object [1-4]. In this case, the subsystems included in the considered set of objects may be unstable [5, 6]. A large number of researchers are engaged in the problems of the sustainable functioning of such systems [7, 8, 9]. The simplest example of an unstable object is an inverted pendulum, a multilink inverted pendulum (inverted two-link pendulum, two wheels inverted pendulum) or a group of inverted pendulums [10-14]. Classical methods of control theory are not always applicable for solving this class of problems.

Designing multivariable automatic control system (MACS) is a complex urgent task $[15,16,17]$. In the paper, the class of homogeneous multivariable automatic control systems of complex technical objects consisting of identical separate subsystems is considered. The systematic method of describing through the characteristics of relations and the characteristics of subsystems for the research is used $[18,19]$. In practice, the tasks of control complex unstable objects functioning as a group are often found. There is offered an approach that allows form the structure and determine the parameters of relations between unstable subsystems to ensure the sustainability of multivariable system.

In the article, the criterion of stability of a homogeneous multivariable automatic control system consisting of statically unstable subsystems is formulated. The efficiency of the developed approach is confirmed by the simulation results for the highlevel systems.

The article poses the problem of synthesizing multivariable automatic control system for unstable multidimensional objects. At the same time, matching the roots of the characteristic polynomial of the system of the control object allows to reduce the order of the system being synthesized and thereby improve its characteristics. The solution of the problem of synthesis is achieved by the method of dynamic decomposition taking into account for the stable part of the characteristic polynomial of the object.

\section{DESCRIPTION OF FREQUENCY METHODS IN \\ SOLVING THE PROBLEM OF SYNTHESIS MACS}

Multivariable automatic control system is a system with multiple inputs and outputs, as well as experiencing the influence of several disturbing forces (fig. 1).

To solve the problem of multiply connected control of subsystems and ensure the stability of this class of multivariable automatic control system, the frequency the method proposed by academician B.N. Petrov and his science school in paper [20] is used.

The description of the multivariable automatic control system is not carried out in a matrix form, but is based on the description of the system through the individual characteristics of the subsystems and the multiply-connected characteristics of communication. As an individual characteristic of a separate subsystem, its transfer function $\Phi_{i}(s)$ in the control mode is considered, when the subsystem functions in a state isolated from other subsystems. 


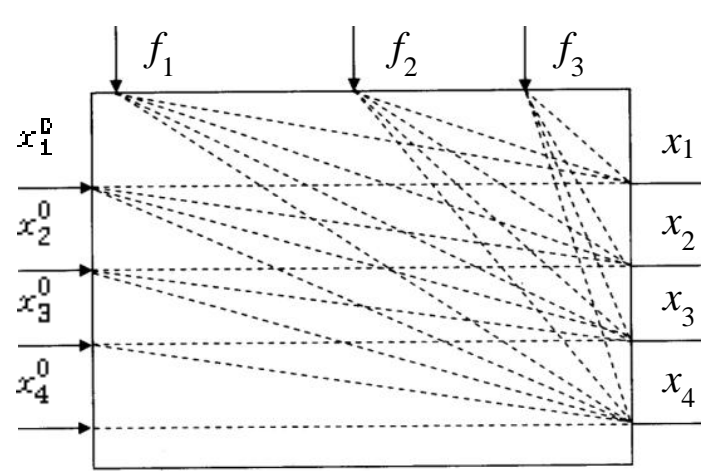

Fig. 1. Structure of MACS

The characteristic equation of a homogeneous multivariable control system with transfer functions $\Phi_{i}(s)=\Phi(s)$, in which the connection between subsystems occurs only through a multiply connected object, or a controller, has the form:

$$
D(\Phi, H)=1+H_{2}(s) \Phi^{2}(s)+\ldots+H_{n}(s) \Phi^{n}(s)=0
$$

where $H_{k}(s), \quad k \in \overline{2, n}$ is the characteristic of communication between the $k$ subsystems in the MACS, consisting of $\mathrm{n}$ subsystems. A detailed calculation of these characteristics is given in [20].

Here is considered the problem of forming a stable multiple-connected system consisting of unstable subsystems, due to the introduction of stabilizing nonholonomic connections between the subsystems. The variety of problem statements is determined by the fact that the subsystems can be:

- stable;

- statically unstable;

- dynamically unstable.

In addition, one part of the subsystems can be statically unstable, the other part - dynamically unstable, and the third part - stable. It is important not to load a stable subsystem, but to distribute the load due to the introduction of compensating connections in solving this problem. Otherwise, the stable subsystem can also leave the stability state and this will complicate the further solution of the stabilization problem.

The aim is to identify the features of multipleconnected control dynamically and statically unstable subsystems and the construction of the procedure for forming links between them from the condition of ensuring the stability of the multiply connected system generally. The problem of forming the necessary and sufficient conditions for the stability of a multiply connected system consisting of statically unstable subsystems is solved.

The task of ensuring the sustainability of management of a complex dynamic system, which includes unstable subsystems, can be solved in two ways:
- due to the coverage of unstable objects with additional feedbacks ensuring their stability, with the subsequent multiply connected control of these new objects;

- due to the organization of nonholonomic stabilizing links between unstable objects.

There is a large number of types of connections between subsystems that depend on real objects and the possibilities of their mutual functioning.

The simplest structure of combining objects into a single whole is a ring structure with one direction of influence of the subsystems on each other (fig. 2).

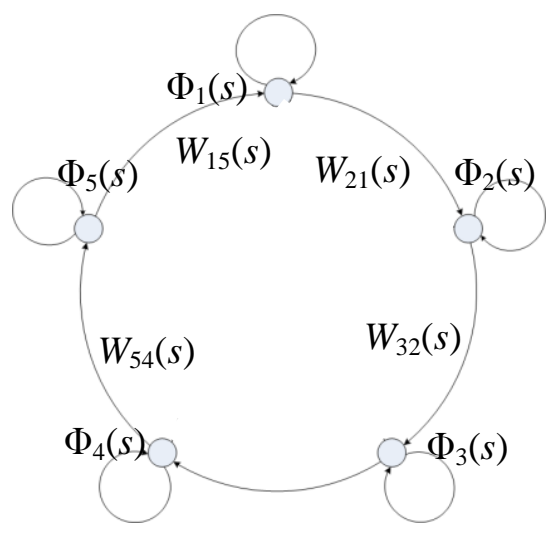

Fig. 2. Combining subsystems into a ring structure

The synthesized link characteristic can be obtained with various cross-linking structures. From the distribution of relationships, the form of the characteristic equation does not change, i.e. The MACS will remain stable, however it affects the quality of the transient processes.

\section{DEVELOPMENT OF THE ALGORITHM FOR CALCULATING RELATIONSHIPS WHEN INTEGRATING UNSTABLE OBJECTS}

Let us analyse the solution of the problem of forming a stable MACS from unstable subsystems due to the introduction of artificial cross-links, and not due to a change in the structure of the subsystems themselves. Consider the formation of a stable doubly connected system consisting of two unstable subsystems with the same individual transfer function, which is equal to

$$
\Phi(s)=\frac{r_{i i}(s) W_{i}(s)}{1+r_{i i}(s) W_{i}(s)}, i=1,2
$$

From practice, it is known that when a signal is fed into a system with a positive feedback, it is necessary to introduce an inverter to preserve the physical meaning of the control signal. The desired characteristic of a two-dimensional bond lets denote $H(s)$. In this case, the communication characteristic is

$$
H(s)=-\frac{r_{21}(s) r_{12}(s)}{r_{11}(s) r_{22}(s)} .
$$


Further, for simplicity, we assume that the links in direct channels equal to 1 . Then the coupling characteristic $H(s)$ will be some polynomial from s.

Thus the characteristic equation of a doubly connected system is:

$$
D(H, \Phi)=1+H(s) \Phi^{2}(s)=0 .
$$

We write the characteristic equation in the form of a polynomial in the variable $s$. Using the Hurwitz stability criterion, we define the structure $H(s)$ in such a way that the resulting system is stable. Consider the problem of synthesizing links between statically unstable subsystems using the method described above.

Example. It is necessary to ensure the joint stable functioning of two statically unstable objects of the first order by introducing a stabilizing bond $H(s)$ between them. The transfer functions of the open subsystems are in the form

$$
W(s)=K / s .
$$

Since objects are covered by a single positive feedback, the transfer functions of closed subsystems have the form

$$
\Phi(s)=1 /(T s-1)
$$

where $T=1 / K$.

The characteristic equation after the introduction of a two-dimensional connection with the characteristic $H(s)$

$$
D(H, s)=T^{2} s^{2}-2 T s+1+H(s)=0,
$$

where $H(s)$ is an unknown function.

It is known that for the stability of a second-order system, it is necessary and sufficient to satisfy the positive condition of all coefficients. It follows that to compensate the instability of objects, the introduction of a two-dimensional first-order coupling is sufficient. Let the communication characteristic be (3):

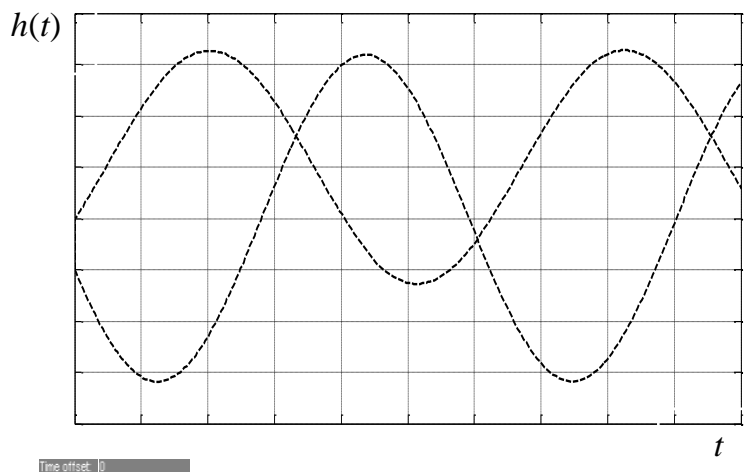

a) $h_{0}=2 T, r_{12} r_{21}=0.5$, MACS on the border of oscillatory stability;

$$
H(s)=\left|\begin{array}{cc}
0 & r_{12}-\tau_{12} s \\
r_{21} & 0
\end{array}\right|=h_{0} s+h_{1},
$$

where $h_{1}=-r_{12} r_{21}$,

$$
h_{0}=\tau_{12} r_{21} \text {. }
$$

Then the characteristic equation (1) in accordance with the adopted transformations is:

$$
T^{2} s^{2}+\left(h_{0}-2 T\right) s+1+h_{1}=0 .
$$

From equation (5) we receive such requirements to the parameters of the system, necessary for the sustainable functioning:

dynamic stability condition:

$$
h_{0}>2 T,
$$

static stability condition:

$$
1+h_{1}>0
$$

When inequalities are fulfilled

$$
1+h_{1}=0, h_{0}=2 T
$$

the system is on the border of oscillatory stability, this is demonstrated by the graphs of the transition process (Fig. 3, a).

When equality is fulfilled

$$
1+h_{1}=0
$$

i.e. coupling coefficients take values

$$
r_{12} r_{21}=1
$$

the system is on the boundary of aperiodic stability. When conditions (6), (7) are fulfilled, the system is stable and controllable (Fig. 3, b) with an accuracy of $\varepsilon=0$ under the action of step signals on the input.

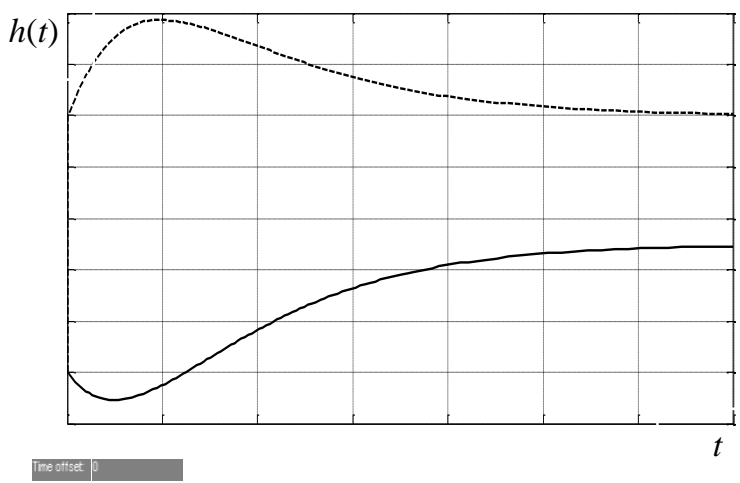

b) $h_{0}=4 T, r_{12} r_{21}=0.5$, system is stable

Fig. 3. Transients in the synthesized system 
When the values of the characteristics of bonds lying outside the found areas, the multivariable system is unstable.

Constructed transient characteristics are insensitive to small changes in the parameters of the subsystems and the synthesized connections between them. Also synthesized links are physically realizable, which has an engineering meaning

Next, we consider the relative location of the hodograph of the amplitude-phase characteristic (APC) of the separate subsystem $\Phi(j \omega)$ and root hodographs $x_{i}(j \omega), \quad i=1,2$ of the characteristic coupling equation when the frequency changes from 0 to $+\infty$ (Fig. 4).

Depending on the speed of movement of hodographs to the origin, various options are possible for the multivariable system.

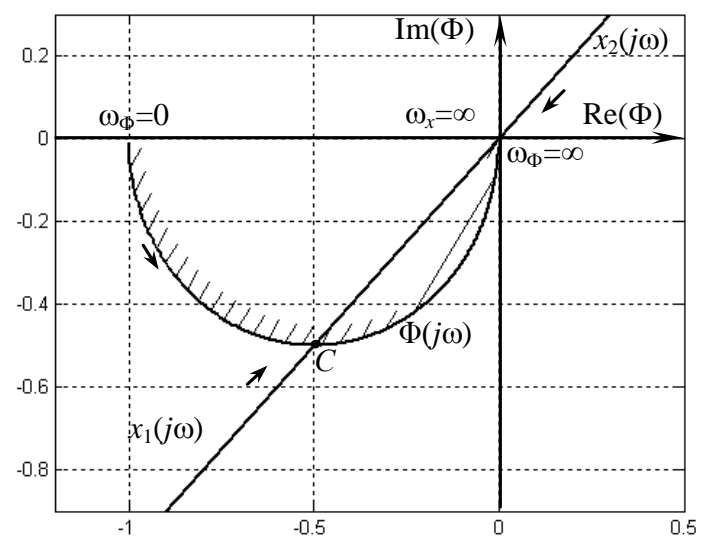

Fig. 4. The mutual arrangement of the hodographs

Consider the intersection point $C$ of the hodographs $\Phi(j \omega)$ and $x_{i}(j \omega), i=1,2$.

At this point, for separate subsystems and connections, there is a balance of amplitudes

$$
|\Phi(j \omega)|=\left|x_{i}(j \omega)\right|, i=1,2
$$

and a balance of phases

$$
\varphi(\Phi(j \omega))=\varphi\left(x_{i}(j \omega)\right), i=1,2 \text {. }
$$

Let denote the frequency of passing the point $C$ of the root godograph $x_{i}(j \omega), i=1,2$ as $\omega_{x}$ and the APC hodograph frequency $\Phi(j \omega)$ of the separate subsystem as $\omega \Phi$. Depending on the values of the coupling coefficients $h_{1}$ and $h_{0}$, the hodographs $\Phi(j \omega)$ and $x_{i}$ $(j \omega), i=1,2$, intersect at different frequencies $\omega x$ and $\omega \Phi$. When $h_{0}=2 T$, the hodograph simultaneously pass through the intersection point $\mathrm{C}$. At the same time, the MACS is on the border of oscillatory stability, which is consistent with previous calculations and simulation results.
Sustainability criterion. For stability of the MACS consisting of statically unstable first-order subsystems, it is necessary and sufficient that the root godograph frequency $\omega x$ and the frequency $\omega \Phi$ of APC unstable subsystem at the intersection point $C$ satisfy the following inequality: $\omega x<\omega \Phi$.

Communication is not physically realizable. In practice, the implementation of this connection can be solved in two ways.

- Replacement of the ideal differentiating link with a real differentiating one. Then the characteristic of the connection, where $\tau<<1$.

- Using the control signal by mistake. For this, the flexible connection $h_{0} s$ is transferred beyond the control object (integrator), which is equal to adding the output coordinate with an improper error.

The use of the above method for determining a stabilizing bond allows us to find the boundary value of the characteristic of bonds.

\section{THE APPlicAtion OF THE AlgORITHM FOR THE SYNTHESIS OF COMPLEX SYSTEM LINKS}

Consider the problem of forming the characteristic of connections for a doubly connected system consisting of statically unstable second-order subsystems.

An example of such a system is a multilink inverted pendulum. The multilink inverted pendulum is a system consisting of statically unstable, connected subsystems. When all the links of the pendulum are the same, the objects is homogeneous. If the links have different physical properties (length, thickness, the material from which they are made, etc.), the objects is heterogeneous.

A model of a system of two identical coupled inverted two-link pendulums with fixed points of suspension $O_{1}$ and $O_{2}$ is shown in Fig. 5. The suspension is carried out with the help of ideal (without friction) cylindrical hinges.

The same hinges at points $D_{1}$ and $D_{2}$ interconnect the links of the inverted pendulum, which are absolutely rigid bodies.

In the fig. 5 such notation is entered:

- $\varphi_{1}$ and $\varphi_{2}$ are counterclockwise angles of deviation from the vertical of the first and second links, respectively;

- $m_{1}$ and $m_{2}$ are the masses of the first and second links; 


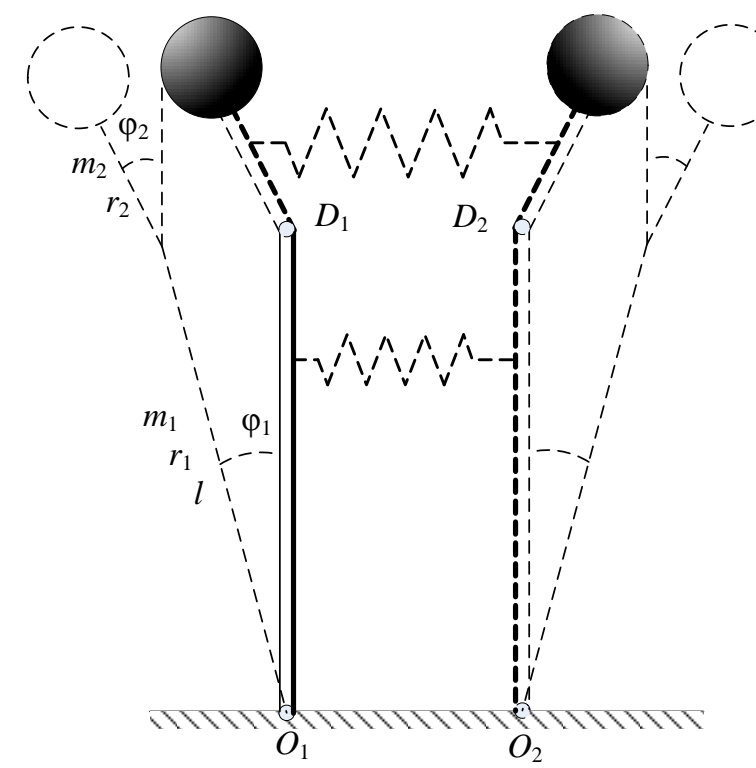

Fig. 5. Dual-link pendulums with fixed points of suspension

- $\quad r_{1}$ and $r_{2}$ are the distances from the hinges $O_{i}$ and $D_{i}$ to the centers of mass of the first and second links, respectively, $i=1,2$;

- $\quad l$ is the length of the first link $O_{i} D_{i}, i=1,2$;

- $k$ is the stiffness coefficient of the springs. Different links of pendulums may have different stiffness coefficients.

The transfer function of the closed separate subsystem is $\Phi(j \omega)$. Rewrite the characteristic equation (1) of the variables $H$ and $\Phi$ as a polynomial of the complex variable $s$.

$D=a_{0}^{2} s^{4}+2 a_{0} a_{1} s^{3}+\left(a_{1}^{2}-2 a_{0}\right) s^{2}-2 a_{1} s+1+H(s)=0 \quad D(H, s)=a_{0}^{2} s^{4}+2 a_{0} a_{1} s^{3}+\left(a_{1}^{2}-2 a_{0}\right) s^{2}-2 a_{1} s+1+H(s)$

For the structural stability of this doubly connected system, it is necessary and sufficient that the characteristic $H(s)$ of the introduced bond is of the second order.

Such a construction $H(s)$ corresponds to a twodimensional connection of the form:

$$
H(s)=\left|\begin{array}{cc}
0 & r_{12}-\tau_{12} s \\
r_{21}+\tau_{21} s & 0
\end{array}\right|=h_{0} s^{2}+h_{1} s+h_{2},
$$

where $h_{0}=\tau_{12} \tau_{21}$,

$$
\begin{aligned}
& h_{1}=r_{21} \tau_{12}-r_{12} \tau_{21}, \\
& h_{2}=-r_{12} r_{21} .
\end{aligned}
$$

Then the characteristic equation of a biconnected corrected system will have the following form:

$$
D(H, s)=a_{0}^{2} s^{4}+2 a_{0} a_{1} s^{3}+\left(a_{1}^{2}-2 a_{0}+h_{0}\right) s^{2}+\ldots
$$

$$
+\left(h_{1}-2 a_{1}\right) s+1+h_{2}=0
$$

Necessary conditions for the stability of the system expressed by inequalities:

$$
\left\{\begin{array}{l}
h_{1}>2 a_{1}, \\
h_{2}>-1, \\
a_{1}^{2}+h_{0}>2 a_{0} .
\end{array}\right.
$$

The necessary and sufficient condition of stability according to the Hurwitz criterion is written as:

$$
2 a_{1}\left(a_{1}^{2}-2 a_{0}+h_{0}\right)\left(h_{1}-2 a_{1}\right)>a_{0}\left[\left(h_{1}-2 a_{1}\right)^{2}+4 a_{1}^{2}\left(1+h_{2}\right)\right\rfloor
$$

As follows from this example, using the reduced method of determining the characteristics of bonds to ensure the stability of a doubly connected system consisting of statically unstable second-order subsystems can be quite laborious. The characteristics of links introduced between statically unstable subsystems to ensure stable operation of the MACS can be determined in another way. Let us consider the solution of the problem of forming a multidimensional characteristic of nonholonomic relations between statically unstable subsystems ensuring their stable joint functioning based on the construction of the desired characteristic equation.

1) Consider the characteristic equation of the multivariable automatic control system, consisting of given statically unstable subsystems with the characteristic connection $H(s)$ : which is obtained with the appropriate transformations expression (1).

2) We form the desired characteristic equation of a stable multivariable control system, for example, consisting of stable subsystems with a transfer function and the desired characteristic of a homogeneous coupling $h_{4}$.

$$
D^{*}(H, s)=a_{0}^{2} s^{4}+2 a_{0} a_{1} s^{3}+\left(a_{1}^{2}+2 a_{0}\right) s^{2}+2 a_{1} s+1+h_{4}
$$

3) Sought for connection characteristic $H(s)$ is determined from the equality to zero of the difference between these equations:

$$
D^{*}(H, s)-D(H, s)=4 a_{0} s^{2}+4 a_{1} s+h_{4}-H(s)=0 .
$$

So the communication characteristic $H(s)$ expressed by the following equality:

$$
H(s)=4 a_{0} s^{2}+4 a_{1} s+h_{4} .
$$


Thus, the connection characteristic depends on the choice of the desired characteristic connection equation, which is given from the stability condition. In this case, it is impossible to indicate the boundary values of the coefficients of the coupling characteristic, i.e. the synthesized system is obviously stable with given stability stocks.

With the passage of time and the development of science and technology, control systems are becoming more and more reliable, and, accordingly, the range of application of unstable objects that are used in practice is expanding.

\section{CONCLUSION}

Managing a group of unstable objects is possible in the mode of both autonomous operation of each element and a unified system. These methods have both advantages and disadvantages. The problem of stabilizing the unstable equilibrium position of a controlled system presents certain difficulties, especially if the resources of the governing bodies are limited. The main results of the paper are as follows.

- The ways of introducing stabilizing bonds are considered depending on the initial objects and the existing relationships between them.

- An approach is proposed that allows the structure to be formed and the parameters of relations between unstable subsystems (objects) to be determined to ensure the stability of a multiply connected system as a whole.

- The stability criterion of a homogeneous MACS consisting of statically unstable subsystems is formulated.

The effectiveness of the developed approach is confirmed by the simulation results. In the future, it is planned to analyse the possibilities of improving the quality of the transient processes of the system with different distributions of connections between objects for the development and production of reliable, accurate and simultaneously high-speed systems [21]. It is necessary to develop software for computer-aided design [22].

\section{ACKNOWLEDGMENT}

The work is supported with the Grants of Russian foundation for basic research № 18-08-00702 A, № 18-08-01299 A.

\section{REFERENCES}

[1] Patil R., Kavitha P., Patra N., Sharma A., Apalani I. Design and development of peristaltic soft robot using shape memory alloy actuators with different control strategies // IOP Conference Series: Materials Science and Engineering. 2018. P. 12044 .

[2] Karimi Z., Khosrowjerdi M.J., Batmani Y. Multiobjective fault-tolerant fixed-order/PID control of multivariable discrete-time linear systems with unmeasured disturbances // Optimal Control Applications and Methods. 2018. Vol. 39. № 5. P. 1648-1662.

[3] Chen C., Liu Z., Xie K., Zhang Y., Wen C., Chen C.L.P. Adaptive asymptotic control of multivariable systems based on a one-parameter estimation approach // Automatica. 2017. Vol. 83. P. 124-132

[4] Juhany K.A.T., Balamesh A.S.A., Kada B. Hybrid high-order sliding mode-based control for multivariable cross-coupling systems: scale-laboratory helicopter system application // Aeronautical Journal. 2017. Vol. 121. № 1243. P. 1319-1341.

[5] Gribkov, V.A., Khokhlov, A.O. Experimental study of inverted regulable pendulum stability // Herald of the Bauman Moscow State Technical University, Series Natural Sciences. 2017. № 2 (71). P. 22-39.

[6] Karthick S., Saran Kumar K., Palani I.A. Fractional order pid control of an underactuated balancing system // 1st IEEE International Conference on Power Electronics, Intelligent Control and Energy Systems, ICPEICES 2016 1, Applications of Control Systems and Power Electronics for Sustainability of Energy Systems. 2016. P. 7853577.

[7] Bui H.-L., Tran D.-T., Vu N.-L. Optimal fuzzy control of an inverted pendulum // Journal of Vibration and Control. 2012. Vol. 18. № 14. P. 2097-2110.

[8] Horibe T., Sakamoto N. Optimal swing up and stabilization control for inverted pendulum via stable manifold method // EEE Transactions on Control Systems Technology. 2018. Vol. 26. № 2. P. 708-715.

[9] Manita L., Ronzhina M. Optimal control of a spherical inverted pendulum // Lobachevskii Journal of Mathematics. 2017. Vol. 38. № 5. P. 954-957.

[10] Kubyshkin V.A., Postnov S.S. Analysis of two optimal control problems for a fractional-order pendulum by the method of moments // Automation and Remote Control. 2015. Vol. 76. № 7. P. 1302-1314.

[11] Ronzhina M.N. Optimal conditions with chattering in the inverted two-link pendulum control problem // Journal of Applied Mathematics and Mechanics. 2016. V. 80. № 1. P. 16-23.

[12] Wang J.-J. Stabilization and tracking control of $\mathrm{x}-\mathrm{z}$ inverted pendulum with sliding-mode control // ISA Transactions ${ }^{\circledR}$. 2012. Vol. 51. № 6. P. 763-770

[13] Octavio Gutiérrez Frias O. Stabilization of the two wheels inverted pendulum by means lyapunov approach // RIAI Revista Iberoamericana de Automatica e Informatica Industrial. 2013. Vol. 10. № 1. P. 30-36.

[14] Ismail J., Liu S. Efficient planning of optimal trajectory for a furuta double pendulum using discrete mechanics and optimal control // IFAC-PapersOnLine 2017. P. 10456-10461.

[15] Stefanescu I.B., Stoica A.M. Control laws for multivariable systems applied in helicopter APCs design // AIP Conference Proceedings (International Conference of Numerical Analysis and Applied Mathematics) ICNAAM 2017" 2018. P. 370008.

[16] Ping Z., Huang J. Global robust output regulation for a class of multivariable systems // International Journal of Robust and Nonlinear Control. 2013. Vol. 23. № 3. P. 241-261.

[17] Xavier N., Bandyopadhyay B., Schmid R. Robust nonovershooting tracking using continuous control for linear multivariable systems // IET Control Theory and Applications. 2018. Vol. 12. № 7. P. 1006-1011.

[18] Ilyasov B.G., Saitova G.A., Khalikova E.A. Management of unstable objects in a multi-connected automatic system // Modern problems of science and education. 2015. № № 1; URL: http://www.science-education.ru/125-19941.

[19] Ilyasov B.G., Saitova G.A., Khalikova E.A. The synthesis of algorithms for intelligent control of gas-compressor unit to ensure the required quality // Modern top technologies. 2018. № 12. Vol. 2. P.271-275.

[20] Ilyasov B.G., Saitova G.A., Khalikova E.A. Analysis of the stability margins of homogeneous multivariable control systems // Journal of Computer and Systems Sciences International. 2009. № 4. P. 4-12.

[21] Churikov Y.V. Quality characteristics of control system // Bulletin of Moscow University of Finance and Law. 2011. № 3. P. 108-117.

[22] Ilyasov B.G., Saitova G.A., Khalikova E.A. Program system for designing of multivariable control system for complex objects // Computer science and information technologies (CSIT'2009): proceedings of the 11th International Workshop. Crete, Greece, 2009. Vol. 1. P.96-100. 